\title{
Donación y trasplante de órganos en medio de la pandemia COVID-19: Consecuencias y retos para un país emergente
}

\section{Organ Donation and Transplantation in the Midst of the COVID-19 Pandemic: Consequences and Challenges for an Emerging Country}

\author{
1 Unidad de Urología, Departamento de Cirugía, Universidad Nacional \\ de Colombia, Hospital Universitario Nacional, Bogotá, Colombia \\ 2 Facultad de Medicina, Universidad de la Sabana, Bogotá, Colombia \\ 3 Unidad de Urología, Fundación Jiménez Díaz, Oficina de Investigación de la \\ Confederación Americana de Urología, Madrid, España \\ ${ }^{4}$ Coordinación de Donación y Trasplantes, Hospital Universitario San \\ Ignacio, Bogotá, Colombia \\ ${ }^{5}$ Coordinación de Donación y Trasplantes, Fundación Clínica Shaio, \\ Bogotá, Colombia \\ 6 Unidad de Urología y Trasplante Renal, Fundación Clínica Shaio, \\ Bogotá, Colombia
}

David Andrés Castañeda-Millán ${ }^{1}$ Julián Pardo-Acuña ${ }^{1}$ David Augusto Cortés-Páez ${ }^{2}$

Ana María Autran-Gómez ${ }^{3}$ Alejandra María Pérez-Pachón ${ }^{4}$ Liz Niño-Ramírez ${ }^{5}$ William Fajardo-Cediel ${ }^{6}$

Address for correspondence Castañeda-Millán David Andrés, MD, Unidad de Urología, Clínicas Quirúrgicas, Hospital Universitario Nacional de Colombia, Bogotá, Colombia

(e-mail: dacastanedam@unal.edu.co).

\section{Resumen}

Palabras clave

- obtención de tejidos y órganos

- trasplante de órganos

- virus del SRAS
La pandemia del SARS-CoV 2 es uno de los eventos en salud pública más relevantes de los últimos años, sus efectos se han traducido en millones de casos de contagios, en más de 430.000 fallecimientos y en un aumento en la necesidad de camas de cuidados intensivos alrededor del mundo. En ese contexto, se ha presentado una reducción significativa de la actividad de donación y trasplantes en varios países, y Colombia no ha sido ajena a ese fenómeno. El presente artículo, pretende dar al lector una perspectiva del estado actual de la donación y trasplantes en el mundo y en Colombia, así como plantear algunos retos que genera la pandemia del SARS-CoV 2 para la ejecución segura del proceso de donación y trasplantes en países con recursos humanos, financieros y de infraestructura limitados.

The SARS-CoV 2 outbreak is one of the most important events of public health around the world; this disease has affected millions of people, has killed over 430.000 people and has increased the needed of intensive care unit beds around the world. During the pandemic the world has seen a decline in the organ donation and transplantation activities, Colombian transplant model has been affected too. This paper wants to show the current situation of organ donation and transplantation during SARS-CoV 2 pandemic and explore some dilemmas around organ donation and transplantation for emerging countries. received

May 25, 2020

accepted

June 26, 2020
DOI https://doi.org/

10.1055/s-0040-1715637.

ISSN 0120-789X.

e ISSN 2027-0119.
Copyright (c) 2020, Sociedad Colombiana License terms de Urología. Publicado por Thieme Revinter Publicações Ltda., Rio de Janeiro, Brazil. Todos los derechos reservados.

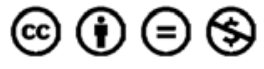




\section{Introducción}

La pandemia del SARS-CoV 2 (Síndrome Respiratorio Agudo y Grave por Coronavirus 2) es uno de los eventos en salud pública con mayor impacto global y sin precedentes en la historia mundial reciente. A la fecha de la elaboración de este escrito, se habían confirmado 7,941.791 casos a nivel mundial y 434,796 fallecimientos asociados a SARS-CoV $2 ;^{1}$ en Colombia se han registrado 53,063 casos, con un total de 1726 fallecimientos y una tasa de letalidad del $3,3 \%{ }^{2}$

La pandemia por infección coronavirus-2019 (COVID-19) ha encaminadoy priorizado los esfuerzos sanitarios mundiales y nacionales hacia las vías de atención hospitalaria para pacientes sintomáticos respiratorios. El impacto de la pandemia en otras esferas asociadas al cuidado crítico se ha traducido en una disminución del número de pacientes con enfermedades crónicas que requieren cuidado intensivo, ${ }^{3}$ una disminución del pool de potenciales donantes cadavéricos de órganos secundaria al descenso de pacientes neurocríticos ${ }^{4} \mathrm{y}$ por ende, un posible estancamiento de pacientes en lista de espera para trasplante de órganos.

La situación actual plantea grandes dilemas y retos para los programas de donación y trasplantes, ya que esos se ven enfrentados a variables que pueden afectar negativamente la seguridad y eficiencia del proceso de atención, dentro de ellas se encuentran:

- El riesgo de adquisición y transmisión cruzada de la enfermedad por COVID-19 en el contexto hospitalario por parte de los equipos de donación humana y trasplante.

- El aumento en los tiempos de gestión de la donación y mantenimiento del donante en muerte cerebral secundarios a la ejecución de protocolos para descartar infección por COVID-19 en los donantes cadavéricos (con las consecuentes repercusiones de la muerte cerebral en la calidad de los injertos).

- El aumento de la ocupación de camas de unidad de cuidados intensivos (con la limitación para el cuidado de donantes en muerte encefálica y receptores de trasplante).

- El riesgo de transmisión del virus donante - receptor o de adquisición del virus por parte del receptor durante su estancia hospitalaria.

- El aumento del riesgo de mortalidad para pacientes en lista de espera secundario a la prolongación del tiempo requerido para acceder al trasplante en áreas donde se deba suspender temporalmente esa actividad.

En ese contexto, es pertinente reflexionar sobre las repercusiones que ha generado la pandemia SARS-CoV 2 en los programas de donación y trasplantes alrededor del mundo y en sus posibles implicaciones para países cuya situación actual está enmarcada en la disponibilidad limitada de recurso humano en salud, de recursos económicos y de infraestructura física. El presente artículo es una reflexión sobre el estado actual de la donación y trasplantes en el contexto de la pandemia COVID-19, sobre su repercusión en la actividad de donación y trasplantes a nivel mundial y nacional, y sobre los retos que genera esta pandemia para la práctica segura de la donación humana y el trasplante de órganos.

\section{Métodos}

Se ejecutó una revisión narrativa de la literatura en las bases de datos Medline, Embase y Lilacs utilizando los términos MeSH: "Tissue and organ donation," "Organ donation," "COVID-19," "Severe acute respiratory syndrome coronavirus 2." Se ejecutó una búsqueda secundaria en revistas científicas especializadas en donación y trasplantes sobre artículos relacionados con el impacto del COVID-19 en esta área y una búsqueda comparativa de datos nacionales en donación y trasplantes durante el periodo enero - mayo de 2019 y enero - mayo de 2020. Se incluyeron en el análisis, los artículos que aportan datos sobre el impacto y los dilemas que ha generado la pandemia COVID-19 en el proceso de donación y trasplantes.

\section{Resultados y Discusión}

Actualmente se sabe que el COVID-19 puede tener una alta tasa de transmisión, con valores de R0 que pueden ser tan altos como 2,68 (IC95\%: 2,47-2,86), ${ }^{5}$ y a pesar de las diferentes medidas de mitigación o supresión ejecutadas en diversas latitudes, esa pandemia ha logrado colapsar la capacidad de respuesta asistencial y la ocupación de las unidades de cuidados intensivos en varias regiones del mundo. Por ejemplo, la región de Lombardía (una de las más afectadas en Italia por la pandemia COVID-19), requirió el aumento en la capacidad instalada de cuidados intensivos de hasta un $90 \%$ para lograr el manejo de los cerca de 1,381 pacientes que requirieron atención en UCI durante el mes de febrero; de forma similar, en la ciudad de Nueva York (Estados Unidos) la tasa de ocupación de las camas y de pacientes ventilados en las UCI entre marzo y abril de 2019 y el mismo periodo en 2020, creció del $64 \%$ y $33,9 \%$ al $78 \%$ y $84,2 \%$, respectivamente. ${ }^{6-8}$

\section{Impacto de la Pandemia COVID-19 en la Donación y Trasplantes: Experiencias Internacionales}

Durante el inicio de la pandemia por COVID-19 en Holanda se registró un descenso del $67 \%$ en el número total de trasplantes ejecutados y se suspendió la actividad de donante vivo. ${ }^{9}$ En Italia durante las primeras cuatro semanas de la pandemia, se registró un descenso entre el $25 \%$ y el 30\% del total de extracciones de órganos para trasplante provenientes de donantes cadavéricos. ${ }^{10}$ España, uno de los países más afectados por la pandemia COVID-19, presentó un descenso cercano al $84 \%$ en la actividad de donación cadavérica y en el número de trasplantes ejecutados tras la declaración de estado de alarma nacional así como el cierre de la actividad de donación de vivo, ${ }^{11}$ allí el promedio de donantes cadavéricos y trasplantes ejecutados pasó de 7,2/día y 16,1/día respectivamente a 1,2/día y 2,1/día durante el periodo de estado de alarma nacional. ${ }^{12}$ En Estados Unidos se registró un cese de actividades en el $71,8 \%$ de programas con donante vivo para trasplante renal y en el $67,7 \%$ para trasplante 
Tabla 1 Efectos de la pandemia SARS-CoV 2 (Síndrome Respiratorio Agudo y Grave por Coronavirus 2) en la actividad de donación y trasplantes de algunos países hasta mayo de 2020. *Dato correspondiente a la regional 1-Red de donación y trasplantes

\begin{tabular}{|c|c|}
\hline Pais & $\begin{array}{l}\text { Donación y trasplante durante } \\
\text { pandemia SARS-CoV } 2\end{array}$ \\
\hline Holanda & $\begin{array}{l}\text { Disminución del } 67 \% \text { en trasplantes } \\
\text { ejecutados y suspensión de trasplantes } \\
\text { con donante vivo }{ }^{9}\end{array}$ \\
\hline Italia & $\begin{array}{l}\text { Disminución del } 30 \% \text { en procuración } \\
\text { de órganos. }{ }^{0}\end{array}$ \\
\hline España & $\begin{array}{l}\text { Disminución del } 84 \% \text { en donación y } \\
\text { trasplante, suspensión temporal de } \\
\text { trasplantes con donante vivo. }{ }^{11,12}\end{array}$ \\
\hline $\begin{array}{l}\text { Estados } \\
\text { Unidos }\end{array}$ & $\begin{array}{l}\text { Disminución del } 51,1 \% \text { en trasplantes } \\
\text { totales y } 71,8 \% \text { en trasplantes con } \\
\text { donante vivo } 13,14\end{array}$ \\
\hline Francia & Disminución del $90,6 \%$ de trasplantes ${ }^{14}$ \\
\hline Colombia & $\begin{array}{l}\text { Disminución del } 63,89 \% \text { de donantes reales, } \\
\text { disminución del 71,05\% en trasplantes } \\
\text { ejecutados*. } 19,20 \\
\text { Disminución del } 56,45 \% \text { de donantes } \\
\text { a nivel nacional } 21,22\end{array}$ \\
\hline
\end{tabular}

hepático. También se estima que aproximadamente el $80 \%$ de programas de trasplante renal con donante cadavérico operan con restricciones mayores incluyendo el trasplante sólo en pacientes altamente sensibilizados con cross-match negativo o con pérdida de acceso vascular para hemodiálisis ${ }^{13}$. Estimaciones recientes, postulan que la actividad de trasplantes con injertos prevenientes de donante cadavérico ha tenido una reducción del 51,1\% en Estados Unidos y 90,6\% en Francia. ${ }^{14}$ (Ver - Tabla 1 )

\section{Donación, trasplantes y COVID-19: ¿Qué ha pasado en Colombia?}

En el año 2018, Colombia registró una tasa de 8 donantes por millón de habitantes, cifra que colocó en ese entonces a nuestro país en el quinto lugar de donación en la región. ${ }^{15}$ Durante el año 2019, se registraron 417 donantes reales y se ejecutaron 1302 trasplantes de órgano sólido, de los cuales 932 fueron trasplantes renales. Pese a esas cifras, a finales del año 2019, se encontraban 3038 personas en lista de espera para trasplante de órgano sólido y se registraron 147 fallecimientos en pacientes incluidos en lista de espera para trasplante. ${ }^{16}$

En el país se confirmó el primer caso de enfermedad por COVID-19 el 6 de marzo de $2020^{17}$ y se decretó el estado de emergencia sanitaria el 12 de marzo de $2020 .{ }^{18}$ Aunque no se dispone aún de datos nacionales validados, en la regional 1 de la Red de Donación y Trasplantes de Colombia, se registraron 13 donantes reales y se ejecutaron 22 trasplantes de órganos sólido durante los meses de marzo, abril y mayo de $2020,{ }^{19}$ esas cifras significan una reducción del $63,89 \%$ y del $71,05 \%$ respecto a los 36 donantes y 76 trasplantes ejecutados en el mismo periodo de tiempo del año 2019. ${ }^{20}$ A nivel nacional se registraron 27 donantes reales en el periodo marzo-abril/2020 comparado con los 62 donantes reales registrados en el mismo periodo de tiempo del año 2019. Eso significa una reducción del $56,45 \%$ en la donación cadavérica en Colombia. ${ }^{21,22}$

El Instituto Nacional de Salud elaboró unos lineamientos técnicos para la donación y trasplantes en época de la pandemia COVID- $19,{ }^{23}$ en ellos se pone de manifiesto la necesidad de pesquisa universal clínica y paraclínica para infección por COVID-19 en cualquier caso de donación (cadavérica o de vivo). Un resultado positivo contraindica el proceso de donación y extracción multiorgánica o el proceso de donación de vivo. También desde las sociedades científicas se han diseñado recomendaciones para el proceso de donación y la atención de pacientes receptores de trasplante renal en contexto de la pandemia COVID-19. ${ }^{24}$ En ellas se reitera que esa población, tiene un riesgo mayor de adquirir la infección por COVID-19 y de generar complicaciones relacionadas con la infección por este virus. El documento recomienda garantizar la ausencia de nexo epidemiológico positivo para COVID-19, disponer de pruebas de certeza (RT-PCR) negativas y de estudios imagenológicos de alta resolución torácicos sin sospecha de infección por COVID-19 para proceder con el proceso de donación (cadavérico o vivo), insta a optimizar el proceso de donación de vivo para trasplante renal con estrategias de aislamiento de contacto estrictas para la pareja donantereceptor durante su estancia hospitalaria, a priorizar procesos de trasplante en pacientes de bajo riesgo inmunológico y a seleccionar donantes cadavéricos con corta estancia hospitalaria y sin criterios expandidos para donación renal.

\section{Dilemas en donación y trasplante durante la pandemia COVID-19}

A pesar de que se ha descrito la infección por COVID-19 principalmente con afectación pulmonar, hasta en el 15\% de casos se ha detectado viremia ${ }^{25}$; y ese fenómeno constituye el sustrato teórico que hace posible la transmisión donante receptor. Actualmente no se conoce con certeza el curso natural y los fenómenos fisiopatológicos generados por la infección por COVID-19, sin embargo, se estima que entre el $14 \%$ y el 53\% de pacientes con SARS CoV-2 tienen lesión hepatocelular inducida por el virus y que en $29 \%$ de pacientes con infección por COVID-19 se detectaron partículas virales en las heces fecales. Esos datos sustentan la no utilización de donantes cadavéricos con COVID-19 para trasplante. ${ }^{26}$ Sin embargo, algunos autores con base en la alta mortalidad de los pacientes en espera de trasplante con falla hepática fulminante o insuficiencia cardíaca avanzada y mortalidad estimada en el corto plazo $>70 \%$ (estados de urgencia inminente o urgencia cero), han planteado la posibilidad del uso de injertos hepáticos y cardíacos provenientes de donantes cadavéricos con COVID-19 asintomáticos o levemente sintomáticos sin repercusiones sistémicas por el virus (cardiomiopatía, falla hepática aguda, falla renal aguda $)^{27}$. Es preciso resaltar que esta postura no ha sido avalada por ningún estudio de alta evidencia científica ni por alguna sociedad internacional de trasplantes. 
Algunos autores consideran que la infraestructura hospitalaria (número de camas UCI, número de ventiladores, disponibilidad de áreas y rutas libres de COVID-19, disponibilidad de hemoderivados, elementos de protección personal para el equipo médico) y la transmisión local del virus, son factores determinantes para la toma de decisiones clínicas en relación a la donación y trasplante; se postula que en términos de racionalizar las intervenciones médicas en medio de la pandemia, es necesario considerar al trasplante renal como una intervención que no salva la vida de un paciente a corto plazo, ya que se cuenta con el soporte dialítico, y por lo tanto podría ser una actividad que se podría posponer sin generar un impacto adverso significativo en el receptor, excepto en casos altamente seleccionados (pérdida de acceso vascular o cavidad peritoneal para diálisis, pacientes altamente sensibilizados). En trasplantes como el hepático, cardíaco o pulmonar, cuyos potenciales receptores tienen un riesgo elevado de mortalidad a corto plazo, la posibilidad de diferir la intervención resulta ser menor y los programas deben instaurar protocolos de atención que permitan minimizar el riesgo de complicaciones asociadas a COVID-19 en caso de continuar con su actividad clínicoquirúrgica, ${ }^{28,29}$ sin embargo, es necesario que en la toma de decisiones clínicas respecto a trasplantar o no, se tomen dichas decisiones con base en la adecuada racionalización y disponibilidad de los recursos humanos y físicos, y en la posibilidad de mitigar riesgos para el equipo de salud y los receptores de trasplante. ${ }^{30}$ Kumar y cols., ${ }^{28}$ plantearon cuatro escenarios para la ejecución de trasplantes de órganos durante la pandemia, con reducciones en la actividad del 25, 50, 75 y 100\% según el estado de urgencia del receptor, la capacidad institucional, la saturación hospitalaria por casos positivos para COVID-19 y la disponibilidad de recurso humano en salud. Ese modelo puede ser de ayuda para la toma de decisiones en las instituciones que brindan servicios de trasplante.

Un consenso internacional reciente recomienda suspender la actividad electiva en trasplantes (trasplante de donante vivo), durante el tiempo de pandemia y minimizar los trasplantes de no urgentes provenientes de donante cadavérico según la dinámica intrahospitalaria (ocupación UCI, disponibilidad de rutas libres de COVID-19); también recomienda considerar la pesquisa para COVID-19 en equipos de procuración de órganos dada la posibilidad de transmisión cruzada del virus, evitar la aceptación de órganos provenientes de donantes de criterios expandidos durante la pandemia, ejecutar pesquisa clínica y con RT- PCR (lavado broncoalveolar o hisopado nasofaríngeo) en donantes cadavéricos, y también recomienda no trasplantar pacientes sintomáticos respiratorios o con infección activa por COVID19. ${ }^{31}$ La sociedad americana de trasplantes recomienda ejecutar una pesquisa clínica en todos los donantes cadavéricos incluyendo la valoración de factores de riesgo o sintomatología asociada a COVID-19 (fiebre, coriza, tos, disnea, dolor abdominal, náuseas, diarrea, ageusia, anosmia) durante los últimos 21 a 28 días previos a la atención hospitalaria además de contar con por lo menos una prueba diagnóstica negativa para COVID-19 en los últimos 3 días previos a la extracción multiorgánica ${ }^{32}$. La organización nacional de trasplantes de España recomienda contar idealmente con prueba microbiológica reciente (RT- $\mathrm{PCR}<24$ horas) negativa para COVID-19 en el donante cadavérico para proceder con el proceso de donación, también recomienda no descartar donantes con historia de infección por COVID-19 curada y tratada, con doble prueba confirmatoria (RT PCR) negativa y con un periodo mínimo de 21 días tras haber superado el cuadro clínico y haber culminado el tratamiento. ${ }^{33}$ Algunos autores plantean que contar con una sola prueba diagnóstica negativa para COVID-19 en el donante cadavérico no constituye un hecho que permita omitir los protocolos para mitigación de riesgo para el personal asistencial, los receptores de trasplante o sus familiares, dadas las limitaciones y variabilidad de la sensibilidad que han demostrado las pruebas diagnósticas ${ }^{34,35}$. En Singapur, por ejemplo, actualmente solicitan tres muestras confirmatorias negativas del tracto respiratorio (RT-PCR) del potencial donante cadavérico para dar inicio al proceso de donación. ${ }^{36}$

Wang y col., recientemente describieron su experiencia de 16 trasplantes de órganos sólidos ( 10 renales, 4 hepáticos y 2 pulmonares) provenientes de donante cadavérico realizados durante la pandemia de SARS-CoV 2 en la provincia de Sichuan, China ${ }^{37}$. Se puede inferir que el éxito alcanzado en esa experiencia radicó en: 1) ausencia clínica, paraclínica, epidemiológica e imagenológica de sospecha de infección por COVID-19 en los donantes cadavéricos aceptados, 2) pesquisa universal para COVID-19 en los posibles receptores, 3) disponibilidad de áreas libres de COVID-19 intrahospitalarias y 4) disponibilidad de protocolos de aislamiento de contacto y cuarentena estrictos para los receptores de trasplante.

En la - Figura 1 proponemos un algoritmo para la toma de decisiones seguras en el proceso de donación y trasplantes durante la pandemia por SARS-CoV 2 en el contexto de países emergentes.

\section{Consideraciones finales}

La pandemia por SARS-CoV 2 ha afectado negativamente la actividad de donación y trasplantes a nivel mundial, y a pesar de que a la fecha de elaboración de este manuscrito la situación de salud pública en Colombia parece no haber alcanzado dimensiones desbordantes, nuestro país también ha sufrido los efectos negativos de la pandemia en su actividad de donación y trasplantes.

En el escenario actual que plantea la pandemia por COVID19 para los países emergentes, se hace necesario ajustar la práctica de donación y trasplantes a las recomendaciones internacionales, a la capacidad de respuesta de los sistemas de salud de cada país, al nivel de saturación hospitalario por COVID-19 y a la magnitud de la transmisión local del virus. Durante la pandemia es necesario ejecutar el proceso de donación y trasplante sólo en casos seleccionados y dentro de un contexto de triple seguridad: para todo el equipo médico involucrado en el proceso de atención del donante o receptor (personal asistencial de cuidados intensivos, coordinación de donación y equipo de procuración/trasplantes), para los 


\section{Potencial donante de órganos cadavérico}

Pesquisa clínica

\begin{tabular}{|l|}
\hline Síntomas: \\
$-¿$ Tiene o tuvo fiebre en los últimos 21 a 28 días previos al ingreso al hospital? \\
$-¿$ Tuvo tos, síntomas respiratorios, ageusia, anosmia o coriza en los últimos 21 a \\
28 días previos al ingreso al hospital? \\
$-¿$ Tiene o tuvo diarrea en los últimos 21 a 28 días previos al ingreso al hospital? \\
\\
Factores de riesgo del donante: \\
$-¿$ Tuvo contacto con pacientes sospechosos o confirmados para COVID-19 en \\
los 21 a 28 días previos al ingreso al hospital? \\
$-¿$ Estuvo o está en un área de alta transmisión local para COVID-19? \\
$-¿$ Está en una UCI respiratoria (atención COVID-19)?
\end{tabular}
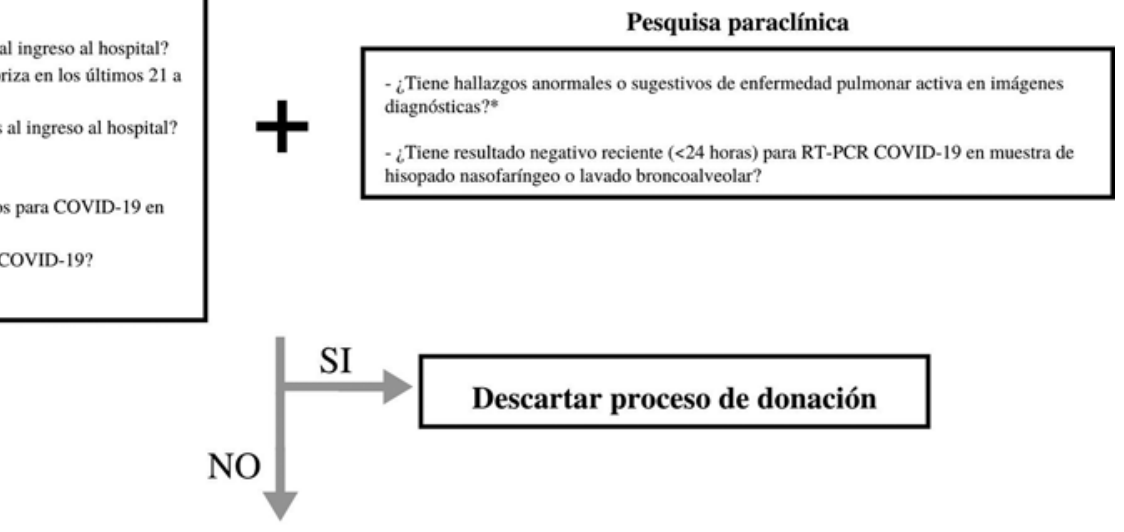

Iniciar proceso de donación

Considerar factores para proceso de donación y trasplante seguro:

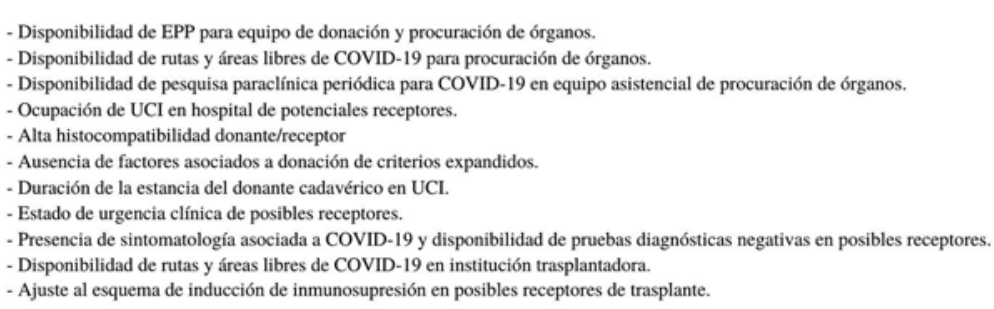

Fig. 1 Algoritmo para la toma de decisiones en donación y trasplante durante la pandemia por COVID-19. *Idealmente tomografía axial computarizada de tórax de alta resolución. COVID-19: Coronavirus 2019. RT-PCR: del inglés Reverse transcription polymerase chain reaction. EPP: elementos de protección personal. UCl: unidad de cuidados intensivos.

posibles donantes y receptores, así como para las instituciones donde se llevan a cabo las distintas fases de este proceso asistencial.

Se desconoce aún del todo la historia natural de la infección por COVID-19 y sus posibles consecuencias para los sistemas de salud de países emergentes, sin embargo, según las cifras descritas, la dinámica que ha presentado la pandemia por SARS-CoV 2 hasta la fecha, las predicciones epidemiológicas de la pandemia y las restricciones actuales para la selección de donantes y posibles receptores, se espera que en el presente año a nivel mundial se evidencie una disminución significativa en términos de donación y trasplante.

\section{Referencias}

1 World Health Organization. Coronavirus disease (COVID-2019) situation reports-148. Disponible en https://www.who.int/ emergencies/diseases/novel-coronavirus-2019/situation-reports / ?gclid=Cj0KCQjw2PP1BRCiARIsAEqv-pQq-ICdE5wnNzZa-rQUq5 jM5HW5n0sKViwHcrU_wSBQHFWbNL-EAulaApn2EALw_wcB [Visitado el 16 de junio de 2020]
2 Instituto Nacional de Salud. COVID-19 Colombia Reporte 15-062020. Disponible en https://www.ins.gov.co/Noticias/Paginas/ Coronavirus.aspx [Visitado el 15 de junio de 2020]

3 Solomon MD, McNulty EJ, Rana JS, et al. The Covid-19 pandemic and the incidence of acute myocardial infarction. N Engl J Med 2020; **: Epub ahead of print. Doi: 10.1056/NEJMc2015630

4 Markus HS, Brainin M. COVID-19 and stroke-A global World Stroke Organization perspective. Int J Stroke 2020;15(04): 361-364. Doi: $10.1177 / 1747493020923472$

$5 \mathrm{Wu}$ JT, Leung K, Leung GM. Nowcasting and forecasting the potential domestic and international spread of the 2019-nCoV outbreak originating in Wuhan, China: a modelling study. Lancet 2020;395(10225):689-697. Doi: 10.1016/S0140-6736(20)30260-9

6 Li R, Rivers C, Tan Q, Murray MB, Toner E, Lipsitch M. Estimated demand for US hospital inpatient and intensive care unit peds for patients with COVID-19 based on comparisons with Wuhan and Guangzhou, China. JAMA Netw Open 2020;3(05):e208297

7 Grasselli G, Pesenti A, Cecconi M. Critical care utilization for the COVID-19 outbreak in Lombardy, Italy: Early experience and forecast during an emergency response. JAMA 2020;323(16): 1545-1546. Doi: 10.1001/jama.2020.4031

8 Badawi O, Liu X, Berman I, et al. Impact of COVID-19 pandemic on severity of illness and resources required during intensive care in the greater New York City area. Medrxiv 2020 
9 de Vries APJ, Alwayn IPJ, Hoek RAS, et al. Immediate impact of COVID-19 on transplant activity in the Netherlands. Transpl Immunol 2020;61:101304. Doi: 10.1016/j.trim.2020.101304

10 Angelico R, Trapani S, Manzia T, et al. The COVID-19 Outbreak in Italy: Initial Implications for Organ Transplantation Programs. Am J Transpl 2020 [Epub ahead of print] . Doi: 10.1111/ajt.15904

11 Organización Nacional de Trasplantes. COVID-19: Impacto en la actividad de donación y trasplantes. Disponible en http://www. ont.es/infesp/Paginas/Impacto_tx.aspx Visitado el 18 de mayo de 2020

12 Domínguez Gil B, Coll E, Fernández Ruiz M, et al. COVID-19 in Spain: Transplantation in the midst of the pandemic. Am J Transpl 2020 [Epub ahead of print] . Doi: 10.1111/ajt.15983

13 Boyarsky B, Po-Yu Chiang T, Werbel W, et al. Early Impact of COVID-19 on Transplant Center Practices and Policies in the United States. Am J Transpl 2020 [Epub ahead of print] . Doi: 10.1111/ajt.15915

14 Loupy A, Aubert O, Reese PP, Bastien O, Bayer F, Jacquelinet C. Organ procurement and transplantation during the COVID-19 pandemic. Lancet 2020;395(10237):e95-e96; Epub ahead of print 10.1016/S0140-6736(20)31040-0

15 Organización Nacional de Trasplantes. Newsletter transplant International figures on donation and transplantation 2018. Disponible en http://www.ont.es/publicaciones/Documents/ NEWSLETTER\%202019_completo\%20integrada\%20cubierta.pdf [Visitado el 12 de mayo de 2020]

16 Coordinación Red Nacional de Donación y Trasplantes - Instituto Nacional de Salud. Informe ejecutivo 2019. Disponible en https:// www.ins.gov.co/Direcciones/RedesSaludPublica/DonacionOrganos YTejidos/Estadisticas/informe-ejecutivo-red-donacion-y-trasplant es-2019.pdf [Visitado el 12 de mayo de 2020]

17 Ministerio de Salud y Protección Social de Colombia. Boletín de prensa 050 de 2020. Disponible en: https://www.minsalud.gov. co/Paginas/Colombia-confirma-su-primer-caso-de-COVID-19. aspx [Visitado el 12 de mayo de 2020]

18 Ministerio de Salud y Protección Social de Colombia. Resolución 385 del 12 de marzo de 2020. Disponible en: https://www. minsalud.gov.co/sites/rid/Lists/BibliotecaDigital/RIDE/DE/DIJ/ resolucion-385-de-2020.pdf [Visitado el 12 de mayo de 2020]

19 Médicos Moduladores de trasplante. Actividad de la Regional 1junio 2020 [Correo electrónico]. 01 de junio de 2020

20 Médicos Moduladores de trasplante. Actividad de la Regional 1 [Correo electrónico]. 24 de junio de 2019

21 Grupo del Centro Regulador de Trasplantes del Instituto Nacional de Salud. Reporte de Actividad Centro Regulador de Trasplantes (CRT) Colombia. Enero - abril 2020. https://www.ins.gov.co/ Direcciones/RedesSaludPublica/DonacionOrganosYTejidos/ Estadisticas/Actividad-Centro\%20Regulador-de-Trasplantes.pdf [Visitado el 12 de mayo de 2020]

22 Grupo del Centro Regulador de Trasplantes del Instituto Nacional de Salud. Reporte de Actividad Centro Regulador de Trasplantes (CRT) Colombia. Junio 2019. Disponible en https://www.ins.gov. co/Direcciones/RedesSaludPublica/DonacionOrganosYTejidos/ Estadisticas/Actividad-Centro\%20Regulador-de-Trasplantes.pdf [Visitado el 12 de mayo de 2020]

23 Instituto Nacional de Salud. Lineamiento técnico para la Red de Donación y Trasplantes relacionados al coronavirus SARS CoV-2, responsable de ocasionar la enfermedad infecciosa por Coronavirus (COVID-19). Disponible en https://www.ins.gov. co/Direcciones/RedesSaludPublica/DonacionOrganosYTejidos/
DocumentosTecnicos/Lineamiento\%20Red\%20Trasplantes\% 20COVID-19\%2015_03_20.pdf [Visitado el 12 de mayo 2020]

24 Serrano R, Cetina C. Benavidez C y cols. Recomendaciones de la Asociación Colombiana de Nefrología en trasplante renal durante la pandemia por coronavirus (COVID-19). Rev Colomb Nefro 2020;7(Suppl 2. Doi: 10.22265/acnef.7.Supl\%202.447

25 Huang C, Wang Y, Li X, et al. Clinical features of patients infected with 2019 novel coronavirus in Wuhan, China. Lancet 2020;395 (10223):497-506. Doi: 10.1016/S0140-6736(20)30183-5

26 Shah MB, Lynch RJ, El-Haddad H, Doby B, Brockmeier D, Goldberg DS. Utilization of deceased donors during a pandemic: argument against using SARS-CoV-2-positive donors. Am J Transplant 2020; 20(07):1795-1799; Epub ahead of print 10.1111/ajt.15969

27 Kates OS, Fisher CE, Rakita RM, Reyes JD, Limaye AP. Use of SARSCoV-2-infected deceased organ donors: Should we always "just say no?" Am J Transplant 2020;20(07):1787-1794; Epub ahead of print 10.1111/ajt.16000

28 Kumar D, Manuel O, Natori Y, et al. COVID-19: A global transplant perspective on successfully navigating a pandemic. Am J Transplant 2020;20(07):1773-1779; Epub ahead of print 10.1111/ajt.15876

29 Martín R, Sierra-Ruiz M, Barrera-Lozano L. Donación de órganos y trasplante en la era COVID-19: ¿Realmente se deben parar los programas? Rev Colomb Cir 2020;35:227-234. Doi: 10.30944/ 20117582.624

30 Wall AE, Pruett T, Stock P, Testa G. Coronavirus disease 2019: Utilizing an ethical framework for rationing absolutely scarce health-care resources in transplant allocation decisions. Am J Transplant 2020; *.*: Epub ahead of print . Doi: 10.1111/ajt.15914

31 Ritschl PV, Nevermann N, Wiering L, et al. Solid organ transplantation programs facing lack of empiric evidence in the COVID-19 pandemic: A By-proxy Society Recommendation Consensus approach. Am J Transplant 2020;20(07):1826-1836; Epub ah ead of print 10.1111/ajt.15933

32 American Society of Transplantation. 2019-nCoV (Coronavirus): Recommendations and guidance for organ donor testing. Disponible en https://www.myast.org/sites/default/files/ COVID19\%20FAQ\%20Donor\%20Testing\%2005.14.2020\%20\%282\% 29.pdf [Visitado el 20 de mayo de 2020]

33 Organización Nacional de Trasplantes. Ministerio de Sanidad Gobierno de España. Infección asociada al nuevo coronavirus (COVID-19). Disponible en http://www.ont.es/infesp/Recomend acionesParaProfesionales/Recomendaciones\%20Donaci\%C3\%B3n \%20y\%20Trasplante\%20frente\%20al\%20COVID-19\%200NT.pdf [Visitado el 20 de mayo de 2020]

34 Woolley AE, Mehra MR. Dilemma of organ donation in transplantation and the COVID-19 pandemic. J Heart Lung Transplant 2020;39(05):410-411. Doi: 10.1016/j.healun.2020.03.017

35 Wang W, Xu Y, Gao R, et al. Detection of SARS-CoV-2 in different types of clinical specimens. JAMA 2020;323(18):1843-1844. Doi: 10.1001/jama.2020.3786

36 Chung SJ, Tan EK, Kee T, et al. Practical considerations for solid organ transplantation during the COVID-19 global outbreak: The experience from Singapore. Transplant Direct 2020;6(06):e554. Doi: 10.1097/TXD.0000000000001002

37 Wang Y, Yang H, Liu H, Buhler LH, Deng S. Strategies to halt 2019 novel coronavirus (SARS-CoV-2) spread for organ transplantation programs at the Sichuan Academy of Medical Science and Sichuan Provincial People's Hospital, China. Am J Transplant 2020;20(07): 1837-1839; Epub ahead of print 10.1111/ajt.15972 